\title{
A note on a stochastic location problem
}

\author{
J.B.G. Frenk \\ Econometric Institute, Erasmus University, Rotterdam, The Netherlands
}

M. Labbé

NFSR Research Associate, C.E.M.E., Université Libre de Bruxelles, Belgium

\section{S. Zhang}

Department of Econometrics, University of Groningen, Groningen, The Netherlands

In this note we give a short and easy proof of the equivalence of Hakimi's one-median problem and the $k$-server-facility-loss median problem as discussed by Chiu and Larson in Computer and Operation Research. The proof makes only use of a stochastic monotonicity result for birth and death processes and the insensitivity of the $\mathrm{M} / \mathrm{G} / k / k$ loss model.

stochastic location; Hakimi median; stochastic monotonicity

\section{Model formulation and results}

In [1] Chiu and Larson consider the so-called $k$-server-single-facility-loss median model $(k$ SFLM). In this model customers at fixed locations generate calls according to a Poisson process with rate $\lambda>0$. If upon arrival of a customer's call at the service facility all its $k$ identical servers are busy this customer is lost at cost $Q>0$. Moreover, if upon arrival some of the servers are idle one of them is assigned to this customer and travels to the location of that customer at a fixed cost rate. The objective is now to determine among a set $\mathscr{F} \subseteq \mathbb{R}^{2}$ of feasible locations that location for the service facility which will minimize the average cost of the system. In order to analyze this model define

- $\mathscr{N}_{i}(\boldsymbol{x}):=$ number of customers being served at time $t$ by one of the $k$ servers if the facility is located at $x \in \mathscr{F}$.

As argued in [1] the queueing process underlying the $k$-SFLM location problem is a $\mathrm{M} / \mathrm{G} / k / k$

Correspondence to: J.B.G. Frenk, Econometric Institute, Erasmus University, Rotterdam, The Netherlands. loss model. For this queueing process it is wellknown (cf. [2]) that for fixed $x \in \mathscr{F}$ the random variable $\mathscr{N}_{t}(\boldsymbol{x})$ converges in distribution to the random variable $\mathscr{N}(\boldsymbol{x})$ representing the number of customers being served in the steady state if $x \in \mathscr{F}$ denotes the location. Moreover, the distribution of $\mathscr{N}(\boldsymbol{x})$ depends only on the arrival rate and the first moment $m(\boldsymbol{x})$ of the service time distribution (Erlang's Loss Formula). If the facility is located at $\boldsymbol{x} \in \mathscr{F}$, the cost function $z(\boldsymbol{x})$ takes the following form (cf. [1]).

$z(\boldsymbol{x})=P_{k}(x) Q+\left(1-P_{k}(\boldsymbol{x})\right) m(x)$

with

- $P_{k}(x):=$ Pricustomer finds upon arrival in steady state all $k$ servers busy $\}=\operatorname{Pr}\{$ customer arriving in steady state is lost\},

- $m(x):=$ expected total travel time of server to arbitrary customer when the facility is located at $\boldsymbol{x}$, and

- $Q:=$ cost per lost customer, $Q \geq 0$.

The main result proved in [1] using lengthy calculations states that the cost function $z(x)$ is increasing in $m(x)$. This implies that the $k$-SFLM location problem is solved by determining the location $x \in \mathscr{F}$ which minimizes the expected 
total travel time $m(\boldsymbol{x})$. Hence, in the special case where $\mathscr{F}$ denotes some network $\mathscr{N}$ this reduces to finding the so-called Hakimi median (cf. [3]) at one of the nodes of $\mathscr{N}$. The above result can be verified easily without any calculations by using a well-known stochastic monotonicity result for birth and death processes. Before proving this we need the following observations. By Little's formula (cf. [5]) the quantity $\left(1-P_{k}(x)\right) m(x)$ equals $(1 / \lambda) L(x)$ where $L(x)$ is the expected number of customers in the system and $\lambda$ is the arrival rate of the Poisson process. Moreover, by the PASTA property, i.e. Poisson Arrivals See Time Averages (cf. [5]), we obtain

$P_{k}(\boldsymbol{x})=\operatorname{Pr}\{\mathscr{N}(\boldsymbol{x})=k\}$

and hence by (1)

$z(\boldsymbol{x})=Q \operatorname{Pr}\{\mathscr{N}(\boldsymbol{x})=k\}+\frac{1}{\lambda} L(\boldsymbol{x})$.

Using (2) one can now prove the following result.

Lemma 1.1. Let $x_{1}, x_{2} \in \mathscr{F}$. Then $m\left(x_{1}\right) \leq m\left(x_{2}\right)$ implies $z\left(\boldsymbol{x}_{1}\right) \leq z\left(\boldsymbol{x}_{2}\right)$.

Proof. Since the expected number $L(x)$ of customers in the steady state equals $\sum_{j=1}^{k} \operatorname{Pr}\{\mathscr{N}(x)$ $\geq j\}$, it is sufficient by (2) to show that $\mathscr{N}\left(\boldsymbol{x}_{1}\right)$ is stochastically smaller than $\mathscr{N}\left(x_{2}\right)\left(\mathscr{N}\left(x_{1}\right) \stackrel{\mathrm{d}}{\leq}\right.$ $\left.\mathscr{N}\left(\boldsymbol{x}_{2}\right)\right)$, i.e. $\operatorname{Pr}\left\{\mathscr{N}\left(\boldsymbol{x}_{1}\right) \geq j\right\} \leq \operatorname{Pr}\left\{\mathscr{N}\left(\boldsymbol{x}_{2}\right) \geq j\right\}$ for every $0 \leq j \leq k$. In order to prove this we observe that for the $\mathrm{M} / \mathrm{G} / k / k$ loss model corresponding to a facility location in $\boldsymbol{x}$ (cf. [2])

$$
\operatorname{Pr}\{\mathscr{N}(\boldsymbol{x})=j\}=\frac{\boldsymbol{\rho}(\boldsymbol{x})^{j} / j !}{\sum_{i=0}^{k} \rho(\boldsymbol{x})^{i} / i !}
$$

for $0 \leq j \leq k$ with $\rho(x)=\lambda m(x)$. This holds in particular for the Markovian loss model with arrival rate $\lambda$ and service rate $1 / m(x)$ and so we are finished by showing that $\mathscr{N}^{(1)} \leq \mathscr{N}^{(2)}$ with $\mathscr{N}^{(i)}$ the number of customers in the steady state in a Markovian $\mathrm{M} / \mathrm{M} / k / k$ loss system $i$ with arrival rate $\lambda$ and service rate $1 / m\left(\boldsymbol{x}_{i}\right)$. If $\mathscr{N}_{t}^{(i)}$ denotes the number of customers at time $t$ in the same Markovian loss system $i$ then the stochastic processes $\left\{\mathscr{N}_{t}^{(i)}, t \geq 0\right\}, i=1,2$, are birth and death processes on the finite state space $\{0,1, \ldots, k\}$ with nonzero transition rates $q_{j, j+1}=$ $\lambda, 0 \leq j \leq k-1$ and $q_{j, j-1}=j / m\left(x_{i}\right)$ for system $i$, $j=1, \ldots, k$. By assumption we know that $m\left(x_{1}\right)$ $\leq m\left(x_{2}\right)$ and hence by a well-known monotonicity result for birth and death processes (cf. Prop. 4.2.10 of [4]) it follows that for every $t>0 \mathscr{N}_{t}^{(1)} \leq$ $\mathscr{N}_{t}^{(2)}$. This implies $\mathscr{N}^{(1)} \stackrel{\mathrm{d}}{\leq} \mathscr{N}^{(2)}$ and so the result is proved.

\section{Acknowledgement}

The authors like to thank the anonymous referee for his useful comments.

\section{References}

[1] S.S. Chiu and R.C. Larson, "Locating an $n$-server facility in a stochastic environment", Comp. Oper. Res. 12, 509-516 (1985).

[2] J.W. Cohen, On Regenerative Processes in Queueing Theory, Lecture notes in economics and mathematical systems, volume 121, Springer-Verlag, Berlin, 1976.

[3] S.L. Hakimi, "Optimal locations of switching centers in a communication network and median of a graph", Oper. Res. 12, 450-459 (1964).

[4] D. Stoyan, Comparison Methods for Queues and other Stochastic Models, Wiley, New York, 1983.

[5] H.C. Tijms, Stochastic modelling and analysis (A computational approach), Wiley, New York, 1986. 